\title{
Ciudad, RuRalidad isleña y políticas PÚBLICAS. EXPERIENCIAS FORMATIVAS DE los jóvenes en el Delta del Paraná (Rosario-Argentina)
}

City, island rurality and public policies. Formative experiences of young people in the Paraná Delta (Rosario, Argentina)

\section{Macarena Romero Acuña}

Conicet / CEACU-UNR, Argentina.

RESUMEN: Este artículo deriva de un proceso de investigación mayor centrado en las experiencias formativas de jóvenes en el pasaje de la escolaridad primaria a la secundaria en el contexto isleño del delta medio del Paraná frente a la ciudad de Rosario Argentina.

Interesa focalizar en las relaciones entre dicha ciudad y el contexto isleño. Se presta atención a las políticas de urbanización, por considerar que las mismas penetran los cotidianos isleños transformando las relaciones laborales de las familias de pescadores. Hipotéticamente se sostiene que dichas transformaciones, inciden en los procesos de escolarización de los jóvenes, en su pasaje de la escuela primaria a la escuela secundaria. En este sentido tales políticas terminan siendo una parte constitutiva de las experiencias formativas que tienen los jóvenes hijos de pescadores. De ahí que a nivel teórico metodológico, estas políticas públicas de urbanización de la ciudad de Rosario, son analizadas desde los procesos isleños.

Palabras clave: políticas públicas, experiencias formativas, Jóvenes, Delta del Paraná, ciudad de Rosario, escuelas de isla. 


\section{ABSTRACT:}

This article draws on a larger research project exploring the formative experiences of young people in their transition from primary to secondary school in the island context of the middle delta of Paraná opposite the city of Rosario, Argentina. It is important to focus on the relations between this city and the island context. Attention is paid to urbanization policies, which affect the daily lives of the islanders by transforming the labor relations of fishing families. We hypothesize that these transformations affect the processes of young people's education in their transition from primary to secondary school. These urban development policies have become a constituent part of the formative experiences of young children from the fishing community. At the methodological-theoretical level, these public urbanization policies in the city of Rosario are analyzed from the perspective of island processes.

KeYWORDS: public policies, training experiences, youth, Delta del Paraná, city of Rosario, island school.

\section{Introducción}

«[...] y acá los de la isla vamos a tener que pedir permiso para subir, para poder ir a Rosario» (Pepeluí, pescador de la Isla Remanso, 9/03/2018)

E ste trabajo (que está en curso) se enmarca en un proyecto doctoral en curso, que cuenta con el financiamiento de una Beca otorgada por el Consejo Nacional de Investigaciones Científicas y Técnicas denominado «Políticas educativas, instituciones y sujetos. Experiencias formativas de jóvenes en el pasaje del primario al secundario en un contexto rural-isleño en Argentina». El tema que propongo se inscribe en el campo de la antropología y educación, y forma parte de un Proyecto de Investigación y Desarrollo ${ }^{1}$

1. PID-SECYT- UNR 2016-2019 (1HUM514): «Políticas Públicas y Cotidianeidad Social. Análisis de experiencias formativas intergeneracionales en contextos urbanos y rurales». 
dirigido por Elena Achilli. El mismo está radicado en el Centro de Estudios Antropológicos en Contextos Urbanos (Rosario, Argentina).

Desde el equipo de investigación en el que me encuentro consideramos que las experiencias educativas (en este caso por la que atraviesan los jóvenes) no son procesos cerrados que sólo se producen o quedan limitados al ámbito escolar (Achilli, 2008). Más bien son parte de experiencias formativas amplias que se configuran en los distintos ámbitos de la vida cotidiana de los sujetos. Es en este sentido que entiendo que el pasaje del primario al secundario no responde únicamente a lógicas escolares y normativas, sino que remiten a la complejidad de la vida social, familiar y laboral de la isla.

Desde esta perspectiva teórica metodológica, entiendo a modo de tesis que ese pasaje de la escolarización primaria a la secundaria, condensa un conjunto de experiencias formativas que se constituyen entre diversas relaciones y condiciones sociales, tanto a nivel de la vida familiar como de la vida escolar. Por lo tanto, el pasaje al secundario (en este contexto de difícil acceso por la falta de este nivel) me permite visualizar la configuración de experiencias que dan cuenta de la interrelación de procesos de distintas escalas. Es decir, procesos a escala de la vida cotidiana de los jóvenes con procesos relacionados a escalas más generales, donde entran en juego, la dimensión de las políticas públicas que generan marcas y orientaciones en la vida familiar y escolar.

Oriento el proceso de investigación desde un enfoque antropológico relacional que combina distintas estrategias desde una lógica intensiva: observación, entrevistas, análisis documental, registro visual. Puntualmente para este artículo, utilizaré los registros de entrevistas realizadas a distintos miembros de familias de pescadores, jóvenes y personal de la escuela primaria pertenecientes a la zona de islas que denominaré Remanso ${ }^{2}$. Además me basaré en el análisis documental de determinadas políticas de urbanización seleccionadas por la modificación en la trama urbana de la ciudad de Rosario, que incidió en la cotidianeidad isleña. Empíricamente la investigación se sitúa en las islas del Delta Medio del Paraná frente a la ciudad de Rosario. Este contexto sufrió en su historia reciente transformaciones socioeconómicas a partir de

2. A fin de respetar el anonimato de los sujetos y las instituciones los nombres que son utilizados son ficticios. 
distintas situaciones: Entre ellas, la finalización de la construcción del puente Rosario-Victoria (2003), los procesos de sojización, la regulación y control de pesca en su comercio nacional e internacional. Sobre estos pueden buscarse avances que se han ido presentado a modo de comunicación en distintos congresos. A su vez también han incidido en las últimas décadas las políticas de planificación urbana de la ciudad: Rosario como ciudad que «mira al río». En este artículo focalizaremos en este último punto, en tanto consideramos que han penetrado en los cotidianos isleños introduciendo nuevas relaciones laborales y de la vida social de las familias de pescadores. Modificaciones que considero, inciden en los procesos de escolarización de los jóvenes y por ende en sus experiencias formativas, tal como desarrollaré más adelante.

Para poder entender cómo tales políticas terminan siendo una parte constitutiva de las experiencias formativas que tienen los jóvenes hijos de pescadores de la zona de Remanso, realizo una presentación del espacio social rural-isleño; luego recuperaré debilidades y fortalezas del Plan Estratégico Rosario Metropolitano (1998; 2008) y el Plan Urbano (2007-2017) por ser las políticas públicas que orientan la recuperación de la zona costera. Finalmente centraré la mirada de estos procesos de urbanización desde la isla, para presentar la complejidad del entramado en la que se tejen las diversas experiencias formativas que viven los jóvenes isleños atravesados por estas políticas públicas.

\section{Los jóvenes y la cotidianeidad en la isla: escuela, familia y trabajo}

«Vivir en la isla es como vivir en el campo, pero con agua» (Dieglo, pescador, 26 años, $\mathrm{RC},{ }^{3}$ 08/01/2016).

«Rosario siempre estuvo cerca» (Paco, pescador, 80 años, isla Remanso,

RC, 11/01/2016)

3. Registro de campo. 
La isla Remanso, tiene características de humedal ${ }^{4}$ que podrían identificarla como selvática. No cuenta con servicios de agua, luz, gas, no hay caminos trazados. Las familias que allí habitan viven en casas de chapas elevadas a 2 metros del nivel del suelo. «Vos le das a un chico de jardín para que te dibuje una casa y lo primero que te hace es la escalera» nos dice el director de la escuela, Pedro en un RC realizado el 28/07/2017. Según los isleños entrevistados, esto se debe a que este material es funcional en época de inundaciones: si se cae una parte de la casa, se puede apuntalar rápidamente; si entra agua a la casa, se puede secar rápido; si hay que mudarse o trasladarse, los materiales permiten con agilidad la venta o movimiento de los insumos de un lado a otro (reconstrucción a raíz de diversos $\mathrm{RC}$ ).

El terreno de la isla Remanso fue expropiado en 1948 a particulares por el Ministerio de Educación de Provincia de Santa Fe, que otorgó la potestad de la entrega de permisos para habitar las tierras a la cooperadora de la escuela, esta información es reconstruida de los Documentos Escolares, Material de Ministerio de Educación de Provincia de Santa Fe). Es en este sentido que hay una suerte de manejo comunal de la tierra: Una vez otorgado el permiso por la cooperadora, la familia elige donde asentarse, construye su vivienda $\mathrm{y}$, en caso de irse puede vender los materiales o llevárselos con ella, pero las tierras siguen siendo potestad de la cooperadora escolar.

Entre los documentos escolares, se pudieron revisar los permisos otorgados y generar una reconstrucción genealógica de la construcción del espacio, es decir, cómo se fueron otorgando desde el año 1948 los permisos por la cooperadora escolar para que las familias fueran asentándose en la isla. Este manejo de la tierra conlleva a que el espacio tenga una lógica de planificación particular: Las familias se van asentando por relaciones de parentesco y se van trazando los caminos entre las casas conforme las mismas se van instalando en el terreno. Pese a esto, hay un camino (que podríamos identificarlo como central) que es el que va a la vera de la línea de estacionamiento de las embarcaciones atravesando las casas y llega hasta la escuela. Sirve para

4. Según la convención de RAMSAR los humedales son ecosistemas que están temporal o permanente inundados y donde el agua es el principal regulador de los ciclos de vida. En muchas entrevistas pescadores nos manifiestan que «lo mejor que nos puede pasar es la inundación, porque el agua limpia y genera nueva vida, la naturaleza se renueva» (Miguel, pescador 45años, RC 06/03/2018). 
hacerse una imagen de cómo es el espacio (que denominaremos rural-isleño, retomando trabajos anteriores) la imagen de una media luna. La parte de la luna que falta completar es una de las lagunas de la zona. La medialuna en sí es el terreno donde se asientan las viviendas, encontrándose la parte más alta en la zona del semicírculo de la luna, donde está la escuela. De la mitad de ese semicírculo del terreno hacia la parte de abajo de la luna, cruza el Paraná grande. De la mitad para arriba encontramos uno de los brazos del río que sigue hasta encontrarse con la laguna, y que es donde los pescadores estacionan sus embarcaciones para resguardarlas en caso de tormentas.

Este espacio social rural-isleño ((Romero Acuña, Calamari, Arce, 2015), que presenta características de ruralidad (pero con la particularidad de ser un contexto inundable y que hemos descrito) tiene 10 hectáreas de territorio y ha sido habitado desde fines de 1800, principios de 1900, principalmente por migrantes andaluces y gitanos según la reconstrucción realizada de la genealogía familiar a los entrevistados. Encontramos así que actualmente hay en la escuela estudiantes que son $7 \mathrm{ma}$ generación de hijos de pescadores de esas familias.

En su cotidianeidad, las aproximadamente 20 familias que viven dentro de estas 10 hectáreas alternan vida, trabajo y estudio con la ciudad de Rosario. Esto se debe a la proximidad: 10 minutos para cruce en lancha, mientras que la ciudad de Victoria Provincia de Entre Ríos, (a la que jurisdiccionalmente pertenecen) queda a $70 \mathrm{~km}$ por vía terrestre.

Actualmente la principal actividad económica de estas familias es la pesca, pero en épocas en la que no puede desarrollarse este trabajo, por veda o por falta de pescados, algunas familias se dedican a la construcción, cuidado de autos o limpieza de casas en la ciudad de Rosario.

Entiendo en este punto que es importante aclarar que quienes habitan este espacio social rural-isleño no son únicamente isleños o pescadores. Hay personas que son de la ciudad de Rosario y tienen emprendimientos económicos, como bares, campings, paradores, que alquilan embarcaciones, disponiendo de servicios gastronómicos y recreativos. Estas personas suelen vivir en la ciudad de Rosario y estar en la isla los fines de semana en temporada baja y vivir en la isla durante la temporada alta. También encontramos gente que vive allí de manera ocasional por dedicarse al cuidado de ganado, vacuno, 
ovino o porcino. En relación a esto, se puede decir que los procesos de sojización llevaron en los últimos años a un desplazamiento del ganado de los campos de la provincia de Entre Ríos a la isla por no ser este un suelo fértil para el cultivo de soja. Este ganado fue trasladado a la isla y con el ganado se trasladaron los peones o puesteros que se encargan de cuidarlo:

[...] fuimos a la isla por una circunstancia de trabajo de él también (refiriéndose a su padre) porque él trabajaba en el campo y el patrón decide en su momento comprar muchos animales para llevar a la isla y necesitaba un peón y bueno». (Lucas, pescador, 22 años, RC, 01/05/2013)

A su vez, hay isleños que van a trabajar a Rosario: «[...] trabajan ahí si son albañiles o han aprendido algún oficio y se defienden bastante bien y trabajan allá en Rosario» (Lucas, pescador, 22 años, RC, 01/05/2013).

También, en los últimos años aumenta la construcción de casas de fin de semana en la zona. Las personas que ocupan estas casas son generalmente personas de la ciudad de Rosario que van también en los fines de semana o en temporada pero no realizan actividades económicas en la zona, razón por la cual algunos isleños que como actividad económica se dedican a trabajar de caseros:

[...] porque la gente que es de acá enfrente viene los fines de semana, [...] o sea, tienen una persona de confianza y entonces le dejan la llave y le cortan el pasto, le abren la casa todo eso, viste que acá en la zona hay lugares hermosos». (Lucas, pescador, 22 años, RC, 01/05/2013)

Este entramado de distintas actividades de la vida socioeconómica y recreativa, da una particularidad a la vida social de la isla, generando dinámicas complejas y que no son lineales. De esta forma, la vida en la isla se va conformando en los entramados de las políticas públicas, las condiciones socio-históricas, y las prácticas y sentidos de la vida laboral de las familias, presentándose realidades que trascienden las dicotomías vida rural y la vida urbana. «La isla es casi como un barrio de Rosario si te pones a pensarlo» (Pedro, director de la escuela, RC, 04/08/2017). Y en estos intersticios de las vidas laborales de las familias, están los jóvenes que debido a estas actividades estacionales de las familias, tienen su matrícula en la escuela Remanso pero el director una o dos veces en el año pide traspaso a alguna escuela de 
la ciudad de Rosario. El traspaso consiste en que los estudiantes son dados de baja en la escuela de la isla para estar habilitados para ir a la escuela en la ciudad. Una vez que vuelven a la isla son dados de baja en la escuela de la ciudad para poder ir a la escuela de la isla.

Como vimos en la delimitación del espacio, la Escuela Remanso tiene una fuerte presencia en la isla. Es una escuela primaria, y pese a que desde el 2006 en Argentina es obligatoria la escolaridad secundaria, la escuela no tiene implementado dicho nivel, aunque se encuentra en tratativas. Concretamente no ahondaré en el desarrollo de este punto, pero sí es importante mencionarlo para pensar cómo esto pone en juego las experiencias formativas de estos jóvenes tanto en términos escolares como a nivel de la misma configuración de lo que supone ser joven/adulto en la isla. En otras palabras, el proceso de escolarización produce un anclaje particular en la vida juvenil pese a estar participando de la vida económica de la familia durante su proceso de escolarización. Vemos que esto pierde continuidad cuando no se logra el pasaje del primario al secundario ya que la tendencia es que los jóvenes dejen de participar del proceso de escolarización y tomen tareas más activas en la economía familiar, esto hace que la vida juvenil se modifique. Como dice Pedro:

La juventud del isleño es muy cortita, porque pasa del nene que va a la escuela a tener que ganarse la vida. [...] es de días porque pasa de ser un chico a ser un adulto que va a ir a remar, a tirar las redes, a criar a un hijo... Jóvenes en edad son. En actitudes ante la vida se convierten en adultos muy rápido. (Pedro, director de la escuela, RC, 04/08/2017)

La falta de continuidad en los estudios secundarios genera una exclusión del sistema ya que el joven no puede ir a la escuela porque no existe este nivel secundario en esta isla, pero a su vez acelera experiencias de la vida juvenil, ya que al terminar el 7 mo grado el estudiante no tiene posibilidad de continuar sus estudios secundarios a menos que cruce a Rosario o se adentre y cruce la laguna para llegar a otra escuela de isla que sí tenga el secundario. Surgen tensiones no sólo entre los jóvenes, sino también para los adultos que reconocen y desean para las generaciones jóvenes la continuidad de los estudios:

El Ministerio está haciendo lo que hace el gobierno... ni saben lo que es acá, piensan en la plata y desde un escritorio, por eso dicen que vayan los chicos a 
Rosario a la secundaria, pero ¿se ponen a pensar qué significa económicamente eso para el pescador? (Pepeluí, pescador, 76 años, RC, 9/03/2018)

En este sentido, es importante retomar discusiones planteadas por Liliana Sinisi y Paula Montesinos respecto de cómo desde 2004 en Argentina se plantean discursos de educación «inclusiva» pero que «encubren un proceso de exclusión» (Sinisi, 1999) y también los reproducen:

[...] yo no tengo título secundario, entonces por eso tengo que limpiar casas [...] podría cruzar con mi marido que trabaja de albañil, él tampoco tiene hecho el secundario y viste, hasta para anotarte de cajera en el súper te piden y acá no es fácil. (Clara, pescadora, madre de 2 estudiantes de la escuela, 24 años, 09/03/2018)

Hasta ahora presenté parte del entramado y conflictividades de la vida cotidiana en la isla y una breve introducción de cómo desde la isla se vive la vida cotidiana en dicho espacio social rural-isleño pero en constante intercambio con la ciudad de Rosario. A continuación, me propongo presentar cómo a través de una política pública la ciudad de Rosario «se mete» en el río; para luego retomar cómo desde la isla «se mira» a esta política pública y como la misma se introduce en la vida cotidiana configurando experiencias formativas.

\section{Rosario una ciudad que «mira al río»}

Antes no había gente más que los que vivían en la isla [...] muy pocos tenían embarcación para cruzar e ir a pasear a la isla... ahora es un libertinaje total $[\ldots]$ Eso cambio porque la ciudad [...] porque la gente empezó a comprar motores [...] y se fue poblando la isla de gente que nunca fue de la isla y que no le interesa más que comer un asado, ir de paseo o turismo [...] No se puede creer lo que es la isla ahora a lo que era la isla cuando fuimos nosotros en el '75 [...] era bastante desolador también te digo, si no tenías a los vecinos de la isla [...] (Pucho, esposo de la ex directora de la escuela Remanso, vivieron 37 años en la isla, RC 15/05/2018) 
La ciudad de Rosario es una ciudad que pertenece a la provincia de Santa Fe (Argentina) y cuenta con 948.312 habitantes, según la proyección del Instituto Nacional de Estadística y Censos (INDEC) realizada sobre el censo nacional del año 2010. Cuenta con 17.869 hectáreas de superficie, es el centro de un área metropolitana (...) que abarca una extensión superior a las 300.000 hectáreas El municipio tiene una longitud de frente costero de $17 \mathrm{~km}$ sobre el río Paraná.

Es una ciudad que históricamente ha desarrollado sus actividades económicas en torno a servicios y actividades relacionadas con la industria automotriz, de refrigeración y la refinería. La zona del Gran Rosario se ha caracterizado por ser sin embargo la que concentra la actividad portuaria, siendo una de las principales zonas agroexportadoras 5 . En este sentido, «el puerto comercial fue la base de la prosperidad económica y social de la ciudad, que se consolidó con la formación del cordón industrial del Gran Rosario» (BID, Mayo 2015: 21).

En este apartado, interesa problematizar cómo a partir de dos políticas públicas de urbanización de la ciudad desarrolladas durante 2007 y 2008 hacen que la ciudad de Rosario pase de «darle la espalda al río» a generar una recuperación de la costa para «mirar al río».

En 1996, se definiría en Rosario las tres dimensiones esenciales de un proyecto estratégico para la ciudad: el diagnóstico de la situación del presente y de los objetivos esperados, la concreción de un proyecto que reuniera al gobierno local con el resto de los actores y que tal proceso sea acompañado por una auténtica participación ciudadana (BORJA, Jordi, 19969. Desde aquí saldría la convocatoria a un conjunto de instituciones públicas y privadas que se plasmaría en la Junta Promotora del Plan Estratégico Rosario (PER). (Stanley 2007: 8)

De esta forma, el Plan Estratégico Rosario Metropolitana de 2008 y el Plan Urbano 2007-2017 son los planes «maestros» que orientan la recuperación de

5. «En Rosario y su región se pueden reconocer varias etapas del desarrollo. Exponente paradigmático del crecimiento económico a partir del modelo primario exportador de fines del siglo XIX y comienzos del XX, en la etapa siguiente, cuando el modelo de acumulación se basó en la producción de manufacturas sustitutiva de importaciones, encontró en Rosario y su región óptimas condiciones para su afianzamiento, así para la década de 1930 el 50\% de las industrias radicadas en la Provincia de Santa Fe se hallaban en Rosario.»(Stanley, 2007: 2) 
la zona costera, definiendo las nuevas características físicas del territorio, así como su funcionalidad y relación con el resto de la ciudad. ${ }^{6}$

Es en ambos planes y a través de concesiones administrativas y convenios urbanísticos, que la Municipalidad consigue montos de inversión (generalmente de privados) y terrenos necesarios para la generación y recuperación del «patrimonio municipal» para el uso público. Esto genera nuevos espacios verdes en la ciudad, que según algunas lecturas como la de Smolka (2013), traen beneficios en términos fiscales por la valoración de espacios recuperados con plusvalías generadas por el proceso de transformación de la ciudad. De esta forma, se utilizan instrumentos urbanísticos de «colaboración público-privada que hacen posible transformar espacios urbanos de vocación pública con inversión del sector privado» (BID, mayo 2015: 19) haciendo financieramente viable toda la operación.

Los planes contemplan un «impacto» de estas políticas de planeamiento no sólo en la ciudad de Rosario si no que plantean cambios en lo que identifican como 3 regiones: Región Metropolitana, Región Centro (Córdoba - Santa Fe) y Región Mercosur (PER 1998, 2008). Es interesante y digno de ser revisado en futuros trabajos qué concepción de «región» plantean los documentos:

El impacto del proceso de globalización y de la revolución científico- técnica, producen cambios contextuales y estructurales en torno al concepto de región. La región es hoy una estructura flexible, cuyos límites no necesariamente deben ser fijados en términos jurisdiccionales- ya que de esta manera, solamente se atendería a uno de sus elementos constitutivos- sino en función de un abanico de aspectos, tales como: el eslabonamiento de procesos productivos,

6. Según datos registrados en PER, los antecedentes de este proyecto se remontan a 1995, año en el que la ciudad de Rosario es incorporada al CIDEU (Centro Iberoamericano de Desarrollo Estratégico Urbano) de Barcelona. Continúa en 1996 con el comienzo de la elaboración del PER, donde en 1998 se lanzan 72 proyectos en 150 instituciones de la ciudad. Respecto de la gestión estratégica del PER en 2002 se realiza una evaluación de un $13 \%$ de avance en el proyecto con un $59 \%$ de grado de avance. Entre 2002 y 2008 se identifican límites político-administrativos para el desarrollo de ciertos proyectos y se empieza trabajar en el planteo de un nuevo «alcance territorial» del PER. En 2008 se realiza un documento denominado PER+10 donde se da por cerrado el PER con un $80 \%$ de los proyectos en funcionamiento o con un avance significativo. En este mismo año se lanza el Plan Estratégico Rosario Metropolitana (PERM). Desde 2007 se ejecuta en la ciudad de Rosario el PUR, que es el Plan Urbano Rosario. $\mathrm{Si}$ bien todas estas políticas públicas tienen distintos objetivos específicos, todos se plantean en sintonía y correlación. 
las articulaciones sociales, los recursos que la rodean, los emprendimientos comunes, las problemáticas concretas a resolver. (PER, 1998: 11)

Resulta interesante retomar la investigación de López Fittipaldi (2017) que indica que los primeros proyectos presentados para ser ejecutados son en relación a obras de infraestructura «que si bien no dependen del municipio, ya que dependen de la jurisdicción nacional, es a él a quien primariamente son funcionales» (López Fittipaldi, 2017: 355). Estas obras son el Túnel Subfluvial y en mayor escala, el Puente Rosario-Victoria, que unen las provincias de Santa Fe y Entre Ríos, «habilitando una ruta directa de integración con el Mercosur y con la región central del país, otra la Hidrovía y por último, aunque muy lentamente, la construcción de la autopista Rosario-Córdoba». (López Fittipaldi, 2017: 355).

En este punto resulta de interés indicar cómo subyacen en las políticas públicas lógicas estructurales que ayudan a la reproducción de las lógicas del capital (trascendiendo inclusive lógicas nacionales) que generan cambios en las condiciones materiales de existencia en la vida cotidiana de los sujetos. Iluminar o preguntarnos respecto de éstas lógicas habilita por un lado trascender las dicotomías micro-macro para pensar en términos de continuidades y rupturas respecto de por ejemplo de qué se habla cuando se hace referencia a «lo regional» (y según quién). Pero por otro lado, permite un análisis en paralelo: «ver cómo en la continuidad que presentan éstos planes, la ciudad es concebida a partir de aquello que deja de ser, para entrever en qué se quiere convertir» (López Fittipaldi, 2017: 355).

Es desde este rastreo que identificamos como continuidad en los planes presentados en la ciudad desde el plan estratégico 1998, que los distintos objetivos se centran en destacar el desarrollo de una gestión pública del territorio, que esté orientada hacia la integración (PER, 2007-2017; 1998; Stanley, 2007).

De este modo, mientras el centro de la ciudad y las áreas costeras se reconvierten para su aprovechamiento por las capas medias, los sectores acomodados y el crecientemente incentivado turismo, se profundizan procesos de reordenamiento espacial que van ubicando a los sectores más desfavorecidos en los márgenes de la ciudad, dando como resultado una «concentración geográfica de la 
pobreza. (Auyero, 2001, citado en Bernardi y Sanchez, 2006, citado en López Fittipaldi 2017)

Es importante destacar que en esta revisión que estamos realizando de las políticas públicas dejamos de lado (por no ser parte del foco de la investigación) la pregunta acerca de qué pasaba en este espacio costero que es «recuperado». Cómo se entramaba esta zona costera «en recuperación» con otros barrios de la ciudad y resulta vital la pregunta: ¿Qué viene sucediendo en barrio Refinería, en la zona de La Florida, en Remanso Valerio, en la zona del Mangrullo? Todos barrios de la ciudad con «acceso» al río. No se ignora que la «recuperación» de este «patrimonio municipal» supuso desalojos, «reordenamiento» de la ciudad, a su vez que supuso uno propuesta de «usos» de estos espacios verdes retomado principalmente por las capas medias. Se reconoce a la vez, que en términos culturales estas políticas públicas dieron accesibilidad a propuestas que trascienden a artistas de renombre para poner en el tablero cultural a bandas, grupos de teatro y distintos artistas locales generando una vasta propuesta cultural de acceso gratuito. Esto en términos de análisis gestión y de del gasto público resulta interesante de ser pensado para entender la dinámica y la conformación de la trama cotidiana de la ciudad de Rosario. ${ }^{7}$

Conforme se van ejecutando los distintos proyectos, se va viendo cómo el Río Paraná va configurándose como parte del espacio social en relación a la ciudad contemplando no sólo el avance y recuperación de la zona costera, sino que también incrementándose la inversión privada en ésta zona que se refleja en la ciudad de una doble forma: en la apertura de espacios públicos verdes que presentan propuestas de uso de la ciudad (recitales y propuestas culturales gratuitas al aire libre, invitación a picnics nocturnos, ferias, entre otros) y en la creación de emprendimientos a través de concesiones (bares, guarderías náuticas, clubes de pesca, servicios gastronómicos, avance del mercado inmobiliario sobre la costa del río). Este «habitar la ciudad» que se va proponiendo e imprimiendo desde las políticas públicas reactiva y genera

7. Sobre esto, se está en conocimiento que hay muchos estudios realizados y distintos centros de estudios que presentan una preocupación por la vida en la ciudad. Sin embargo, por estar mirando estas políticas de la ciudad desde la isla no se ha generado una revisión exhaustiva del material más que la citada en este apartado. 
en los ciudadanos rosarinos nuevos usos y propuestas públicas y culturales en esos espacios verdes; Pero miradas desde la isla, estas propuestas significan el cierre del acceso a la costa de la ciudad., como nos expresan Pancho y veremos en el apartado que continúa también Pepeluí. Sobre esto ahondaremos en el apartado que continúa, que nos permitirá a su vez comprender cómo se configuran las experiencias formativas de los y las jóvenes entre la isla, la ciudad, la vida familiar, laboral y escolar.

\section{A modo de cierre: Las políticas urbanas en las experiencias formativas de los jóvenes isleños}

«Primero la ciudad, el avance. Acá yo te cuento hace 60 años que estoy en la isla $[\ldots]$ vos atracabas ahí en la costa y subías por cualquier lado: subías por Génova, subías por cualquier calle. Ahora tenes que subir por la bajada Génova

y tenes que andar así $[\ldots]$ para poder subir $[\ldots]$ te cerraron todos clubes y cerraron toda la costa. Uno va y dice: yo soy pescador. Pero ¿cuánta gente hay ahí que tienen lancha, que pagan el club, que consumen en bares? Y entonces ¿va a estar molestando un pescador a tanta cantidad de lancha, de cosas que pueden hacer plata ahí?» (Pepeluí, pescador de la Isla Remanso, 76 años, RC,

9/03/2018)

Interesa en este apartado poder dar cuenta de aquellos procesos a escala de la vida cotidiana de los jóvenes en relación con procesos a escalas más generales, donde entran en juego, la dimensión de las políticas públicas que van generando marcas y orientaciones en la vida familiar, escolar y barrial. Para esto, es necesario explicitar que dichas orientaciones se constituyen entre diversas relaciones y condiciones sociales y materiales de existencia, tanto a nivel de la vida familiar como de la vida escolar, constituyendo experiencias formativas intergeneracionales, categoría analítica que comprendemos como:

[...] aquellas prácticas que los sujetos desarrollan a lo largo de su vida en ámbitos vinculados a su cotidianidad. Esos ámbitos abarcan una variedad de instituciones y espacios, desde la escuela, la familia, el trabajo, grupos políticos, la iglesia, etc.' (Espoturno, 2017). Estas 'tienen una temporalidad que es doble: una histórica y que remite a procesos que si bien constituyen al sujeto lo trascienden en su historia 'para atrás', generando sentidos que se anclan en 
las prácticas y experiencias que se viven en lo cotidiano. Pero también, hay una temporalidad que tiene que ver con un sentido «de futuro» (Romero Acuña, 2017). De este modo, abordar las experiencias formativas supone pensar a la educación en un sentido amplio que no remite exclusivamente al ámbito de lo escolar. (Espoturno, Romero Acuña y Pavesio, 2018: 2 y 3)

Se torna vital en nuestro trabajo poder generar una reconstrucción de dichas experiencias formativas en el momento del pasaje del primario al secundario de éstos jóvenes isleños ya que se entiende que este momento:

[...] en un sentido amplio, como experiencia formativa en sí, que permite dar cuenta de cómo en un periodo de tiempo acotado se condensan relaciones familiares, escolares y barriales que dan cuenta de distintos sentidos y expectativas respecto de la escolarización. Estos sentidos son intergeneracionales y ponen en juego distintos valores otorgados a nociones de estudio, trabajo y perspectivas de futuro que tienen por un lado los jóvenes que están transitando el pasaje; pero por otro lado los adultos que acompañan este proceso. Hay una conducción por parte de los adultos a cargo (docentes y familiares responsables) pero también una conducción por parte del Estado, que con determinadas políticas, planes y programas efectiviza la obligatoriedad de este pasaje y finalización de la escuela secundaria. ((Romero Acuña, 2017: 7)

Entendemos que en los últimos años se han realizado diversas revisiones de la categoría de pasaje (Diez, Montesinos y Paoletta 2018; Gessaghi, López, Rúa y Ziliani, 2018; Gómez, Hirsch y Neufeld; 2018) pero interesa volver sobre esta idea de «conducción» por parte del Estado, para pensar concretamente las tramas que se entretejen entre estas experiencias formativas de los jóvenes isleños, sus vidas cotidianas en la isla y la vida en la ciudad.

Las condiciones de acceso a la ciudad; la obligatoriedad que se imprime de la escolarización secundaria y la falta de escuela de dicha modalidad en las 10 hectáreas; sumado a las modificaciones ambientales producto en parte de obras de infraestructura generadas en el río, en parte por uso indiscriminado que se traducen en consecuencias laborales para los pescadores (cambios de circuitos de pesca) y al creciente turismo en esta zona de islas, ha generado cambios en las últimas décadas del espacio social rural-isleño: "Yo te cuento que hace 20 años atrás había 60 o 70 familias viviendo acá, en toda esta zona de acá... y ahora no se si llegamos a 20 familias en la isla» (Pepeluí, pescador 
de la Isla Remanso, 9/03/2018). «Se fueron a Rosario cansados de la creciente y la otra es por el asunto del trabajo. Consiguieron trabajo y eso es lo que es más difícil acá...» (Raquel, cocinera de la Escuela Camalote, 57 años RC 19/03/2018).

Es así que en los últimos, 2 años 5 estudiantes dejaron de asistir a la Escuela Camalote ${ }^{8}$. El primero en irse fue Suso de 11 años. Sus padres están separados desde que él es chico, su papá trabaja en una fábrica en la ciudad donde vive con 2 hermanos de Suso; y su mamá es pescadora y vive en la Isla Remanso con dos hermanos más chicos que Suso que van a Nivel inicial y primaria en la Escuela Remanso. A mitad del sexto grado de escuela primaria Suso decide mudarse a Rosario con su papá y continuar allí sus estudios. En entrevistas informales realizadas luego de su traslado a la ciudad nos comenta que le gusta ir a la escuela en Rosario, que es más difícil en contenidos, pero que allí hay mayor cantidad de «pibes» de su edad y que eso «esta bueno». No extraña «tanto» la vida en la isla porque va los fines de semana, los pescadores lo conocen y siempre alguien lo cruza y entonces puede visitar a sus hermanitos y a su abuela (cocinera de la escuela) y su abuelo.

En el año 2017 terminan el séptimo grado Tina y Ana. Luego de varias reuniones de padres y directivos de la Escuela Remanso con Ministerio de Educación, se efectiviza la propuesta de una beca para traslado a la ciudad de 1000 pesos argentinos mensuales para cruzar el río e ir a una escuela Técnica privada ubicada en la zona Norte de la ciudad de Rosario que también otorga becas a las estudiantes para realizar allí sus estudios.

Para poder entender las decisiones y distintos recorridos de estas estudiantes en proceso de pasaje es necesario remitirse a la realidad familiar de cada una de ellas. Ana es hija única, sus padres no viven en la isla Remanso, sino en la Isla Cañito, sin embargo realizó toda su escolaridad primaria en la Escuela Remanso. Viven allí porque sus padres son encargados del cuidado del predio de uno de los clubes náuticos de la ciudad de Rosario que licitó y compro hectáreas de isla para abrir allí una sede para sus socios. La familia

8. Las reconstrucciones que se realizan a continuación son producto de una síntesis de distintos RC generados en los últimos dos años de trabajo de campo. La mayoría responden a entrevistas realizadas o notas de campo generadas en distintas estancias de intercambio con los jóvenes y sus familias. 
vive en el predio del club en una casa y hace 10 años tienen este trabajo. Ana durante toda su escolarización primaria en días de semana iba a la escuela y en fines de semana de temporada alta ayudaba con las tareas laborales familiares. Desde muy pequeña estuvo en contacto permanente con gente de la ciudad, incluso por ser hija única nos comentaba que tenía muchas amigas «del club» que en época de temporada alta invitaba a quedarse a dormir en la casa, porque los padres al día siguiente volvían al club. En diversos registros manifestó interés por hacer sus estudios secundarios en la isla, pero con la intención de luego ir a Rosario a estudiar Medicina. Hoy sabemos que en las mañanas el padre la cruza en lancha a la ciudad de Rosario tres veces a la semana donde realiza doble turno y los otros días se lleva a la casa tarea para actualizar y mantener el ritmo de sus compañeros de cursada. Tina tiene 5 hermanos y viene de una familia de pescadores que viven en la Isla Remanso. Ella es la penúltima de sus hermanos y en distintas entrevistas comentó cómo se encargaba de las distintas tareas de la casa, de los hermanos y de las compras en momentos que los padres no estaban. Atravesada por condiciones materiales de vida de bajos recursos y problemas de alcoholismo en la familia, Tina transitó su escuela primaria con algunas dificultades, finalizando estos estudios a los 15 años. Llamaba la atención cómo en actos escolares o actividades barriales siempre estaba más cercana a las mujeres o madres de los estudiantes de la escuela que a sus compañeros. Era reconocida entre sus pares por ser una muy talentosa pescadora y en su perspectiva a futuro ella se ve realizando esta actividad económica como modo de sustento. Tina no cruza a la escuela de Rosario; ella va a la escuela secundaria que hay en Isla Cañito. Esta «elección» tiene que ver con que a esta escuela puede llegar remando con su canoa. En algunos días sus padres salen a pescar en la madrugada (4 o 5 de la mañana) y eso imposibilita el cruce del canal con embarcación a motor y genera una incompatibilidad entre la vida laboral de la familia y los formatos de horarios escolares en la ciudad. Por otro lado Tina aún no tiene edad legal para poder cruzar sola con embarcación a motor y aunque así la tuviera lo que le cobrarían por amarrar su embarcación en la costa de Rosario no alcanzaría a ser cubierto ni en 15 días por el valor de la beca que le dan por traslado. Con Tina, pidió el traslado de escuela su hermano menor que iniciaba en 2018 el cursado de su 5to año en la Escuela Remanso, ahora ambos están matriculados en la escuela de Isla Cañito. 
Finalmente, el estudiante Meridiem que iniciaba el 6to grado en 2018. Su padre es pescador y vivían con su familia en la Isla Remanso; tiene un alto compromiso y participación en la escuela y el barrio, es tesorero de la cooperadora escolar. Tuvieron varios «achaques» económicos (dificultades) en los últimos años. La pesca no estaba siendo redituable («el pescado no sale») y las «changas» en la ciudad ya no estaban rindiendo ni siquiera para cubrir el traslado. Consigue trabajo estable en una fábrica ubicada en la zona del Gran Rosario y se trasladan con toda la familia a vivir a la zona sur de la ciudad. En general a la Escuela Remanso le cuesta esta mudanza porque se hace más difícil la firma de rendición de cuentas que mensualmente exige el Ministerio, pero pese a la mudanza, las relaciones de la familia con la escuela y la Isla Remanso permanecen.

Como vemos, las realidades de la ciudad de Rosario y la Isla Remanso se entraman y entrecruzan generando una multiplicidad de relaciones e intercambios sociales, económicos, laborales y escolares. Resulta entonces de particular interés romper con las dicotomías micro-macro, rural-urbano para poder detectar cómo las lógicas homogeneizantes del capital generan transformaciones estructurales que permean la vida cotidiana de los sujetos, atravesándolos y generando no sólo diversas condiciones de existencia, sino también constituyendo experiencias formativas que van sedimentando los diversos recorridos que constituyen. Reconocer cómo esas tendencias del capital se expresan o toman lugar en las políticas públicas se torna imprescindible para comprender un determinado espacio social y las experiencias formativas que allí se entraman. 


\section{Bibliografía}

ACHILLI, E. (2008) Investigación y Formación docente. Editorial Laborde, Rosario.

BID, 2015 http://www.rosario.gob.ar/ArchivosWeb/bid.pdf

Diez, Montesinos y Paoletta (2018) La «relación entre niveles educativos» en el marco de la obligatoriedad escolar: tensiones y complejidades entre las iniciativas estatales y las tramas locales en IV Seminario taller red de Investigación en Antropología y Educación (RIAE). Córdoba, abril 2018 - Tradiciones etnográficas latinoamericanas en educación: interpelaciones y disputas teórico metodológicas en las últimas décadas.

Espoturno, Romero Acuña y Pavesio (2018) Intervenir desde un enfoque socio-antropológico. Un análisis de procesos de investigación en torno a las experiencias formativas intergeneracionales en IV Seminario taller red de Investigación en Antropología y Educación (RIAE). Córdoba, abril 2018 - Tradiciones etnográficas latinoamericanas en educación: interpelaciones y disputas teórico metodológicas en las últimas décadas.

Gessaghi, LóPez, Rúa y ZiLIani (2018) El problema del «pasaje» de la primaria a la secundaria: entre la heterogeneidad de prácticas y los objetivos consensuados en IV Seminario taller red de Investigación en Antropología y Educación (RIAE). Córdoba, abril 2018 - Tradiciones etnográficas latinoamericanas en educación: interpelaciones y disputas teórico metodológicas en las últimas décadas.

Gómez, Hirsch y Neufeld (2018) «Los talleres»: desafíos metodológicos, cambios en las condiciones de producción y prácticas de investigación en IV Seminario taller red de Investigación en Antropología y Educación (RIAE). Córdoba, abril 2018 - Tradiciones etnográficas latinoamericanas en educación: interpelaciones y disputas teórico metodológicas en las últimas décadas.

Ley de Educación NaCional Nº 26.2006 año 2006

López FitTiPaldi, M. (2017) Derecho a la ciudad y una experiencia educativa popular. Revista Iluminuras; Lugar: Porto Alegre; vol. 18 p. 350 368.

PER, 1998, 2008

PERM, 2007-2017

RAMSAR, https://www.ramsar.org/es/node/5696

Romero Acuña, M. (2017) El estudio y los días en la isla: Una aproximación 
antropológica a los sentidos de lo escolar de los jóvenes isleños. xxxi Congreso Asociación Latinoamericana de Sociología. Las encrucijadas abiertas de América Latina. La sociología en tiempos de cambio. Montevideo, 3 a 8 de Diciembre 2017. ISBN en trámite

Romero Acuña, Macarena, Calamari, Mirna y Arce, Itatí (2015) La escuela un lugar de encuentro: Acerca de las prácticas docentes en contexto isleño. Presentado en III Seminario-Taller Red de Investogación en Antropología y Educación (RIAE) Políticas y transformaciones educativas Perspectivas etnográficas en América Latina. Universidad de Buenos Aires - Facultad de Filosofía y letras. Instituto de Ciencias Antropológicas - Sección de Antropología Social. http://ica.institutos.filo.uba.ar/seanso/pae/boletin/index.html

SinISI, L. (1999) La relación nosotros-otros en espacios escolares 'multiculturales'. Estigma, estereotipo y racialización. En: M. R. Neufeld y J. Thisted (Comps.). «De eso no se habla...» los usos de la diversidad sociocultural en la escuela. Buenos Aires: Eudeba. pp. 189-234.

Sмolka (2013) Implementación de la recuperación de plusvalías en América Latina. Políticas e instrumentos para el desarrollo urbano. Editora: Lincoln Institute of Land Policy. ISBN 978-1-55844-293-1

Stanley (2007) Transformaciones económicas en la segunda mitad del siglo xx: problemas y desafos em las ciudades latinoamericanas. Presentado en IV Jornadas Uruguayas de Historia Económica. Montevideo.

Sitios web:

https://publications.iadb.org/handle/11319/6930?locale-attribute=es

http://www.rosario.com.ar/puertoros.htm

http://www.rosario.com.ar/hidro2.htm

http://www.rosario.gov.ar/web/ 